\title{
Deterioration Prediction of Urban Bridges on Network Level Using Markov-Chain Model
}

\author{
Li Li, ${ }^{1,2}$ Lijun Sun, ${ }^{1}$ and Guobao Ning ${ }^{3}$ \\ ${ }^{1}$ Key Laboratory of Road and Traffic Engineering of the Ministry of Education, Tongji University, Shanghai 201804, China \\ 2 Jiangxi Ganyue Expressway Co., Ltd. Nanchang 330025, China \\ ${ }^{3}$ School of Automotive Studies, Tongji University, Shanghai 201804, China
}

Correspondence should be addressed to Guobao Ning; guobao.ning@tongji.edu.cn

Received 28 November 2013; Accepted 20 January 2014; Published 2 March 2014

Academic Editor: Baozhen Yao

Copyright (C) $2014 \mathrm{Li} \mathrm{Li}$ et al. This is an open access article distributed under the Creative Commons Attribution License, which permits unrestricted use, distribution, and reproduction in any medium, provided the original work is properly cited.

\begin{abstract}
Bridges play an important role in urban transportation network. However, it is hard to predict the bridge deterioration precisely in Shanghai, because records of various bridge types with different maintenance status coexist in the same database and the bridge age span is also large. Therefore a Markov-chain model capable of considering maintenance factors is proposed in this study. Three deterioration circumstances are modeled including natural decay, conventional recoverable decay, and enhanced recoverable decay. Three components as well as the whole bridge are predicted including bridge deck system, superstructure, and substructure. The Markov-chain model proposed can predict not only the distribution of the percentage of different condition rating (CR) grades on network level in any year but also the deterioration tendency of single bridge with any state. Bridge data records of ten years were used to verify the model and also to find the deterioration tendency of urban bridges in Shanghai. The results show that the bridge conditions would drop rapidly if no recoverable repair treatments were conducted. Proper repair could slow down the deterioration speed. The enhanced recoverable repair could significantly mitigate the deterioration process and even raise the CR grades after several years of maintenance and repair.
\end{abstract}

\section{Introduction}

An urban bridge management system (BMS) has been put into use in Shanghai since 2002 and become steady since 2004. More than ten years of bridge condition records have been accumulated up to now. Most of the records are about small and medium bridges which also belong to categories II to $\mathrm{V}$ (Table 1) according to Chinese technical code of urban bridges management CJJ 99-2003 [1]. Many types of bridges are included in this BMS database including concrete girder bridges, arch bridges, and steel bridges, where the concrete girder bridges take the majority of the entire data records (more than $80 \%$ ). The bridge maintenance efforts are also in various forms including routine maintenance, minor repair, medium repair, major repair, and even reconstruction. New bridges and old bridges coexist in the same database and the age of some bridges is even over 100 years. The basic condition of the bridges in Shanghai BMS is so complicated that a prediction approach capable of considering a variety of factors should be used to forecast the urban bridge deterioration process.

Two categories of methods can be used to develop bridge deterioration models, deterministic and stochastic methods. The deterministic model assumes that the bridge deterioration tendency is certain, so regression analysis is commonly used to determine the decay rate [2-4]. These models are often relatively convenient to use but fail to consider uncertainty and randomness of the bridge deterioration process [5]. Stochastic models are better in such aspects. Engineering experience can be used to describe the uncertain factors [6]. Discrete probabilistic models based on state and time are effective in predicting the infrastructure facility deterioration [7]. The discrete probability models are represented by Markov process, which is based on the concept of probabilistic cumulative damage [8] and now commonly used in performance prediction of infrastructure facilities [9]. It is believed that Markov process has three advantages [10]. Firstly, it is able to reflect uncertainties of 
TABLE 1: Urban bridge management system in China.

\begin{tabular}{ll}
\hline Category & \multicolumn{1}{c}{ Significance } \\
\hline I & $\begin{array}{l}\text { Super large bridges and bridges } \\
\text { with special structures }\end{array}$ \\
II & Bridges on freeways \\
III & Bridges on arterial roads \\
IV & Bridges on sub arterial roads \\
V & Bridges on bypass \\
\hline
\end{tabular}

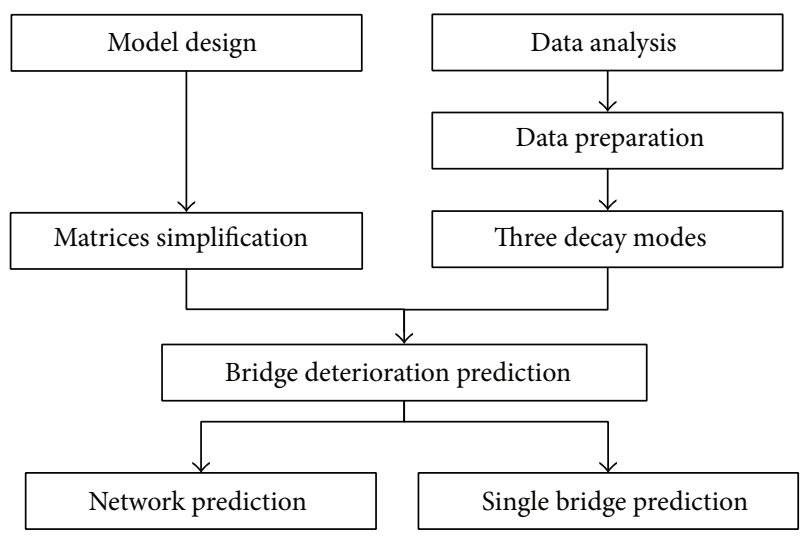

FIgURE 1: The organization of this study.

various aspects [11]. Secondly, the prediction of future state is based on the present state so the model is incremental [7]. And lastly, it can be applied on network level with many facilities involved which requires calculation efficiency [12]. Some bridge management systems have adopted Markov model to predict the performance of bridges such as Pontis and BRIDGIT $[13,14]$.

Although the Markov process has been widely used in bridge performance prediction, most applications only focus on some bridge component such as deck or directly consider the bridge as a whole. Besides, the sample size is small in most applications [15]. Actually every bridge component as well as the whole bridge has different deterioration characteristics and the maintenance status usually has varying degrees of impact on different bridge components. Therefore it is necessary to develop a Markov model based on large sample to predict the deterioration tendency of different components of urban bridges, which is also the objective of this study.

The study is organized as shown in Figure 1 and the paper can be divided into six sections. Section 2 presents the basic form of the Markov-chain model used for bridge deterioration prediction. Section 3 provides details of data records in Shanghai BMS and the data processing principles under two circumstances as well as the corresponding processing results. The calculation of Markov transition probability matrices is in Section 4 and the bridge deterioration prediction using these transition probability matrices is discussed in Section 5. Lastly, the conclusions are presented in Section 6.

\section{Markov-Chain Model}

A Markov process describes a system that can be in one of several states. Each state can pass to another at each time step according to fixed probabilities. Markov-chain model is a special case of the Markov process whose time and state parameters are both discrete. A Markov chain can be treated as a series of state transitions based on certain probabilities. A stochastic process whose transition probability of a future state depends only on the present state is defined as a first-order Markov process [16]. For a stochastic process $\{X(t), t \in T\}$, if the conditional probability can be expressed as follows, $\{X(t), t \in T\}$ is a Markov chain with discrete parameters:

$$
\begin{gathered}
P\left(X_{t+1}=i_{t+1} \mid X_{0}=i_{0}, X_{1}=i_{1}, \ldots, X_{t}=i_{t}\right) \\
=P\left(X_{t+1}=i_{t+1} \mid X_{t}=i_{t}\right)
\end{gathered}
$$

where, $i_{t}$ is the process state at time $t$, and $P$ is the conditional probability of a future event. There are two assumptions about Markov chains. First, the future state of a stochastic process depends only on the present state and has nothing to do with the past. Second, the transition probability between two states should be constant. So the time step needs to be determined properly to keep simple state transition.

Bridge condition decays with time (year) and can be considered as discrete condition states at certain time intervals. Thus Markov chains could be the proper tool to model the bridge deterioration process. As the bridge condition is usually evaluated through several rating levels, the transition probabilities should be expressed as a matrix, called the transition probability matrix. A typical transition probability matrix $P$ is shown in

$$
\begin{aligned}
P= & {\left[\left(\begin{array}{ccc}
p_{11} & \cdots & p_{1 n} \\
\vdots & \ddots & \vdots \\
p_{n 1} & \cdots & p_{n n}
\end{array}\right)\right], } \\
& p_{i j} \geq 0 \quad i, j \in I, \\
& \sum_{j \in I} p_{i j}=1 \quad i \in I,
\end{aligned}
$$

where, $n$ is the number of bridge condition states; matrix element $p_{i j}$ represents the probability that the bridge condition will pass from state $i$ to state $j$ during a certain time step. Therefore, if the initial bridge condition is known, the future condition after $t$ time intervals can be obtained by (3), where $C$ is the condition vector [17]:

$$
C(t)=C(0) \times P^{t}
$$

The transition probability matrix is the key of the Markovchain model and is commonly obtained by statistical data of bridge conditions. Two methods can be used to calculate transition probability matrix, the regression method based on nonlinear optimization $[15,18]$ and the percentage method [19]. The regression method is affected significantly by the prior maintenance actions, whose records may not be readily 
available, while the percentage method requires at least two consecutive condition records without any maintenance interventions [10], and it will generally increase the difficulty of data processing. However, the requirement of the percentage method is comparatively easy to achieve, so it will be used in this paper.

\section{Condition Data on Urban Bridges}

3.1. Data Profiles. An urban bridge management system (BMS) has been put into use in Shanghai since 2002 and become steady since 2004, which means the process of bridge data collection has been standardized and thus the data reliability is improved. Bridge detection is conducted once a year. Therefore there are ten years of bridge condition records available in the BMS up to now. The number of detected bridges increases year by year in most cases with the expansion of detection range. However, quantity decrease may happen occasionally due to bridge demolition, repair, and management change.

Urban bridges are divided into five categories in China according to their significance in the road system [1] as shown in Table 1. Shanghai BMS includes bridges from category II to $\mathrm{V}$ and the bridges of type I are usually under the management of specialized agencies. Thus bridges in Shanghai BMS are almost small and medium bridges, and the concrete girder bridges take the majority of the entire database. Data profiles of bridge records in Shanghai BMS from 2004 to 2013 can be found in Table 2. For bridge deterioration prediction on network level, all types of bridges should be taken into account, so bridge categories will not be considered when calculating the transition probability matrix. But it should be noted that the deterioration tendency predicted in this paper may actually be more meaningful for concrete girder bridges because of the large proportion of such bridge type in BMS records.

3.2. Data Preparation. The bridge condition in Shanghai BMS can be evaluated into five grades as shown in Table 3. On the circumstance of routine maintenance and minor repair, the condition rating $(\mathrm{CR})$ of a bridge should maintain the original grade or decay to the next grade between two consecutive years. In other words CR grade cannot decay beyond one grade on such circumstance. However, in the cases of medium or major repair and reconstruction, the bridge condition may be improved and thus the CR grade can be raised to any level according to the repair efforts. Besides, there might be some detection errors in the BMS which lead to unreasonable CR grades. All these situations coexist in the BMS database which make the deterioration prediction complicated and inaccurate. Therefore the bridge condition data needs to be preprocessed to calculate the transition probability matrix.

Two kinds of deterioration process can be modeled using Shanghai BMS database, natural decay under routine maintenance or minor repair and conventional recoverable decay with medium or major repair as well as reconstruction.
So the data processing methods should be different for each circumstance as follows.

(i) Natural decay: CR grades A to D cannot decay beyond one grade between two consecutive years, while grade E should not change because the bridge condition cannot be even worse. Data records which do not meet the conditions above should be excluded from the BMS database.

(ii) Conventional recoverable decay: $\mathrm{CR}$ grades $\mathrm{A}$ to $\mathrm{C}$ cannot decay beyond one grade between two consecutive years, because only routine maintenance and minor repair will be applied on such bridges. However, CR grades D and E have the chances of being improved because of the repair. So only data records with grades $\mathrm{A}$ to $\mathrm{C}$ need to be processed.

The bridge structure consists of three parts, bridge deck system, superstructure, and substructure. Each part can be evaluated separately and the CR grade for the whole bridge can be obtained by summing the weighted grades of all three parts [1]. Thus a single bridge actually has four data records and all these records should be preprocessed according to the principles above. The result of data preparation is shown in Table 4. Quantity of the valid data records for bridge deck system is the smallest, which is probably because the deck system detection based on visual observation is with the greatest variability. On the contrary, the detection for substructure is comparatively the steadiest, which means the decay of the substructure is slow or the components are hard to detect.

\section{Calculation of Markov Transition Probability Matrices}

4.1. Model Assumption and Matrices Simplification. The CR in Shanghai BMS has five grades which can generate the state vector space of Markov-chain model. For ease of calculation, the state vector should be transformed from $[A, B, C, D, E]$ to $[5,4,3,2,1]$, and elements of the state vector are called status value. As urban bridges in Shanghai are detected once a year, a one-year transition probability could be determined to help simplify the transition probability matrix. Therefore, the probabilities for two (or more) state jumps in two years should be negligible. Besides, the rows of the transition matrix must sum up to one.

It follows that only five transition probabilities are needed to fully define a particular transition matrix in the circumstance of natural decay. The case of the conventional recoverable decay is a little more complicated, but for grades A to $\mathrm{C}$ (also 5 to 3 ) only three transition probabilities are needed. The simplified transition probability matrices are shown in (4), where $P_{\text {nat }}$ is the transition probability matrix 
TABLE 2: Data profiles of bridge records in Shanghai BMS (2004-2013).

\begin{tabular}{|c|c|c|c|c|c|c|c|}
\hline \multirow{2}{*}{ Year } & \multicolumn{7}{|c|}{ Bridge quantity } \\
\hline & Concrete girder & Arch & Steel & Truss & Combined system & Others & Total \\
\hline 2004 & 894 & 129 & 23 & 19 & 26 & 0 & 1091 \\
\hline 2005 & 1139 & 140 & 9 & 14 & 13 & 42 & 1357 \\
\hline 2006 & 1241 & 138 & 8 & 10 & 11 & 39 & 1447 \\
\hline 2007 & 1274 & 142 & 8 & 10 & 11 & 41 & 1486 \\
\hline 2008 & 1404 & 152 & 4 & 9 & 10 & 29 & 1608 \\
\hline 2009 & 1381 & 145 & 10 & 9 & 9 & 36 & 1590 \\
\hline 2010 & 1476 & 142 & 9 & 11 & 9 & 39 & 1686 \\
\hline 2011 & 1521 & 136 & 8 & 11 & 9 & 44 & 1729 \\
\hline 2012 & 1571 & 121 & 7 & 9 & 0 & 48 & 1756 \\
\hline 2013 & 1639 & 86 & 10 & 9 & 0 & 57 & 1801 \\
\hline
\end{tabular}

TABLE 3: Definition of bridge condition ratings.

\begin{tabular}{lcl}
\hline Grade & Definition & Maintenance recommendations \\
\hline A & Intact & Routine maintenance \\
B & Good & $\begin{array}{l}\text { Routine maintenance and minor } \\
\text { repair }\end{array}$ \\
C & Up to standard & Minor repair \\
D & Bad & Medium or major repair \\
E & Dangerous & Major repair or reconstruction \\
\hline
\end{tabular}

under natural decay and $P_{\text {rec }}$ is under the conventional recoverable decay:

$$
\begin{aligned}
P_{\text {nat }} & =\left[\begin{array}{ccccc}
p_{55} & 1-p_{55} & & & \\
& p_{44} & 1-p_{44} & & \\
& & p_{33} & 1-p_{33} & \\
& & & p_{22} & 1-p_{22} \\
& & & & 1
\end{array}\right], \\
P_{\text {rec }} & =\left[\begin{array}{ccccc}
p_{55} & 1-p_{55} & & & \\
& p_{44} & 1-p_{44} & \\
& & p_{33} & 1-p_{33} \\
p_{25} & p_{24} & p_{23} & p_{22} & p_{21} \\
p_{15} & p_{14} & p_{13} & p_{12} & p_{11}
\end{array}\right] .
\end{aligned}
$$

4.2. Transition Probability Matrices Calculation. Valid CR grades of urban bridge records in Shanghai BMS during 2004 to 2013 are used to calculate the transition probability matrix, which have been preprocessed in Section 3.2.

In the circumstance of natural decay, only five transition probabilities are needed for each part of the bridge as well as the whole bridge. The calculation results can be found in Tables 5, 6, 7, and 8. Every two consecutive years have a group of transition probabilities. However, the final transition probability matrix under natural decay should be only one for each part of the bridge as well as the whole bridge. Therefore the calculation results need to be further processed.

As shown by Table 5 to Table 8 , the distribution of transition probabilities for each grade is not uniform. Thus the average value and standard deviation can be used to analyze these data. The transition probabilities that exceed the valid range should be excluded, and the final transition probabilities are the average value of the valid values, as shown in

$$
\begin{array}{r}
R_{i}=\bar{p}_{i i} \pm s_{i} \quad p_{i i}=\overline{p_{i i}^{\text {valid }}} p_{i i}^{\text {valid }} \in R_{i} \\
i \in[5,4,3,2],
\end{array}
$$

where, $R_{i}$ is the valid range of transition probabilities for each grade $i ; \bar{p}_{i i}$ and $s_{i}$ are the average value and standard deviation of transition probabilities through all years for each grade $i ; p_{i i}$ is the final transition probabilities for each grade $i$; $p_{i i}^{\text {valid }}$ is the valid probabilities for each grade $i$. The data processing results can be found in Table 9.

The case for the conventional recoverable decay is a little more complicated because the probabilities of CR grades D and $\mathrm{E}$ for two (or more) state jumps in two years should be taken into consideration. The actual data in Shanghai BMS are used so the transition probabilities of grades D and $\mathrm{E}$ are based on the real situation of urban bridges in Shanghai. The transition probabilities for grades $\mathrm{A}$ to $\mathrm{C}$ are the same as the probabilities under natural decay. The results of grades $\mathrm{D}$ and E are shown in Table 10, where the final probabilities are the average value of the transition probabilities through all years because the assumption that the rows of the transition matrix must sum up to one needs to be satisfied.

\section{Deterioration Prediction Using Markov Chains}

5.1. Condition Rating Prediction on Network Level. With the Markov transition probability matrices and present status of urban bridges, the future status of bridge conditions at any year can be predicted. The transition matrix under natural decay represents the developing tendency that only routine maintenance and minor repair are conducted, while the transition matrix under conventional recoverable decay means all the maintenance and repair treatments are taken into account, which is also the actual situation of urban bridge management situation in Shanghai. But in the light of the technical regulation for urban bridge management [1], all the bridges with CR grade E should be repaired and bridges with CR grade $\mathrm{D}$ should be partly repaired according 
TABLE 4: Number of valid data records.

\begin{tabular}{lcccccccccc}
\hline & & \multicolumn{9}{c}{ Time interval } \\
Decay process & Bridge components & 2004 & 2005 & 2006 & 2007 & 2008 & 2009 & 2010 & 2011 & 2012 \\
& & 2005 & 2006 & 2007 & 2008 & 2009 & 2010 & 2011 & 2012 & 2013 \\
\hline \multirow{3}{*}{ Decay 1 } & Deck system & 627 & 859 & 879 & 1058 & 1227 & 1161 & 1153 & 1276 & 1366 \\
& Superstructure & 785 & 1094 & 1149 & 1227 & 1359 & 1386 & 1393 & 1410 & 1361 \\
& Substructure & 866 & 1133 & 1185 & 1309 & 1391 & 1480 & 1449 & 1499 & 1565 \\
\hline \multirow{3}{*}{ Decay 2 } & Deck system & 700 & 1036 & 973 & 1140 & 1252 & 1199 & 1224 & 1311 & 1461 \\
& Superstructure & 834 & 1132 & 1186 & 1275 & 1375 & 1397 & 1414 & 1442 & 1396 \\
& Substructure & 877 & 1144 & 1191 & 1313 & 1392 & 1481 & 1456 & 1502 & 1567 \\
\hline
\end{tabular}

Decay 1: natural decay; Decay 2: conventional recoverable decay.

TABle 5: Transition probabilities for bridge deck system under natural decay.

\begin{tabular}{lcccc}
\hline Year & $p_{55}$ & $p_{44}$ & $p_{33}$ & $p_{22}$ \\
\hline $2004-2005$ & 0.782 & 0.563 & 0.614 & 0.755 \\
$2005-2006$ & 0.886 & 0.699 & 0.799 & 0.905 \\
$2006-2007$ & 0.884 & 0.687 & 0.710 & 0.805 \\
$2007-2008$ & 0.861 & 0.662 & 0.857 & 0.828 \\
$2008-2009$ & 0.843 & 0.593 & 0.712 & 0.963 \\
$2009-2010$ & 0.820 & 0.667 & 0.847 & 0.902 \\
$2010-2011$ & 0.902 & 0.676 & 0.861 & 0.920 \\
$2011-2012$ & 0.901 & 0.660 & 0.808 & 0.919 \\
$2012-2013$ & 0.763 & 0.640 & 0.765 & 0.667 \\
\hline
\end{tabular}

TABLE 6: Transition probabilities for superstructure under natural decay.

\begin{tabular}{lcccc}
\hline Year & $p_{55}$ & $p_{44}$ & $p_{33}$ & $p_{22}$ \\
\hline $2004-2005$ & 0.949 & 0.828 & 0.727 & 0.857 \\
$2005-2006$ & 0.964 & 0.872 & 0.818 & 0.813 \\
$2006-2007$ & 0.921 & 0.730 & 0.750 & 0.818 \\
$2007-2008$ & 0.938 & 0.896 & 1.000 & 0.846 \\
$2008-2009$ & 0.952 & 0.842 & 0.786 & 1.000 \\
$2009-2010$ & 0.961 & 0.712 & 0.789 & 0.909 \\
$2010-2011$ & 0.927 & 0.698 & 0.500 & 0.647 \\
$2011-2012$ & 0.955 & 0.778 & 0.952 & 0.833 \\
$2012-2013$ & 0.854 & 0.702 & 0.849 & 0.692 \\
\hline
\end{tabular}

to their damage degree. However, these requirements are hardly fully realized in actual practice due to the budget and manpower limits. In this study an ideal status called "enhanced recoverable repair" assumes that all "E bridges" (bridges with CR grade E) and most of the "D bridges" are repaired. Furthermore most of the repaired bridges can recover to the top level. This assumption is mainly based on the technical regulation for urban bridge management [1] and the current management status of urban bridges in Shanghai. According to the regulation [1], all "E bridges" and parts of "D bridges" need to be repaired, but neither the maintenance level nor the maintenance proportion are clearly defined. Besides, based on the current technical capacity, financial resources, and management mechanism of the urban bridge
TABLE 7: Transition probabilities for substructure under natural decay.

\begin{tabular}{lcccc}
\hline Year & $p_{55}$ & $p_{44}$ & $p_{33}$ & $p_{22}$ \\
\hline $2004-2005$ & 0.931 & 0.803 & 0.692 & 1.000 \\
$2005-2006$ & 0.957 & 0.899 & 1.000 & 1.000 \\
$2006-2007$ & 0.936 & 0.841 & 1.000 & 1.000 \\
$2007-2008$ & 0.932 & 0.922 & 1.000 & 1.000 \\
$2008-2009$ & 0.966 & 0.890 & 1.000 & 1.000 \\
$2009-2010$ & 0.947 & 0.936 & 0.875 & 1.000 \\
$2010-2011$ & 0.951 & 0.895 & 0.750 & 0.000 \\
$2011-2012$ & 0.949 & 0.909 & 1.000 & 0.500 \\
$2012-2013$ & 0.881 & 0.904 & 0.786 & 1.000 \\
\hline
\end{tabular}

TABLE 8: Transition probabilities for whole bridge under natural decay.

\begin{tabular}{lcccc}
\hline Year & $p_{55}$ & $p_{44}$ & $p_{33}$ & $p_{22}$ \\
\hline $2004-2005$ & 0.887 & 0.855 & 0.944 & 0.905 \\
$2005-2006$ & 0.947 & 0.876 & 0.903 & 0.929 \\
$2006-2007$ & 0.921 & 0.859 & 0.815 & 0.958 \\
$2007-2008$ & 0.918 & 0.921 & 0.889 & 1.000 \\
$2008-2009$ & 0.949 & 0.954 & 1.000 & 1.000 \\
$2009-2010$ & 0.929 & 0.883 & 0.909 & 0.909 \\
$2010-2011$ & 0.909 & 0.836 & 0.750 & 1.000 \\
$2011-2012$ & 0.912 & 0.917 & 0.911 & 0.867 \\
$2012-2013$ & 0.796 & 0.835 & 0.951 & 0.889 \\
\hline
\end{tabular}

management in Shanghai, only parts of the "D and E bridges" can be effectively repaired, and the maintenance level is also uncertain. Therefore, the ideal maintenance status should fully comply with the requirement of the regulation and be provided with enough budget and technical support. As a result of such assumption, all "E bridges" and at least half of the "D bridges" should be repaired to the top CR level. The probabilities for "D bridges" of maintaining the original state and being degraded refer to the current practical status of "D bridges." The transition probabilities under such status are shown in Table 10, and all bridges components as well as the whole bridge are assumed to have the same transition probabilities for "D and E bridges." 
TABLE 9: Final transition probabilities under natural decay.

\begin{tabular}{lcccc}
\hline Category & Element & Average value & Standard deviation & Final probability \\
\hline \multirow{3}{*}{ Bride deck system } & $p_{55}$ & 0.849 & 0.048 & 0.859 \\
& $p_{44}$ & 0.650 & 0.042 & 0.665 \\
& $p_{33}$ & 0.775 & 0.079 & 0.773 \\
& $p_{22}$ & 0.851 & 0.032 & 0.880 \\
Superstructure & $p_{55}$ & 0.936 & 0.073 & 0.946 \\
& $p_{44}$ & 0.784 & 0.135 & 0.778 \\
& $p_{33}$ & 0.797 & 0.099 & 0.787 \\
Substructure & $p_{22}$ & 0.824 & 0.023 & 0.943 \\
& $p_{55}$ & 0.939 & 0.039 & 0.120 \\
& $p_{44}$ & 0.889 & 0.333 & 0.952 \\
Whole bridge & $p_{33}$ & 0.900 & 0.043 & 0.938 \\
& $p_{22}$ & 0.833 & 0.039 & 0.921 \\
& $p_{55}$ & 0.907 & 0.070 & 0.878 \\
& $p_{44}$ & 0.882 & 0.049 & 0.925 \\
\hline
\end{tabular}

TABLE 10: Final transition probabilities under conventional recoverable decay.

\begin{tabular}{lccccc}
\hline Element & Bridge deck system & Superstructure & Substructure & Whole bridge & Enhanced recoverable decay \\
\hline$p_{25}$ & 0.216 & 0.178 & 0.155 & 0.303 & 0.500 \\
$p_{24}$ & 0.122 & 0.090 & 0.104 & 0.074 & 0 \\
$p_{23}$ & 0.253 & 0.273 & 0.275 & 0.102 & 0 \\
$p_{22}$ & 0.355 & 0.374 & 0.415 & 0.488 & 0.400 \\
$p_{21}$ & 0.055 & 0.085 & 0.051 & 0.034 & 0.100 \\
$p_{15}$ & 0.131 & 0.280 & 0.278 & 0.474 & 0 \\
$p_{14}$ & 0.087 & 0.066 & 0.037 & 0.032 & 0 \\
$p_{13}$ & 0.145 & 0.124 & 0.167 & 0.032 & 0 \\
$p_{12}$ & 0.268 & 0.164 & 0.148 & 0.111 & 0 \\
$p_{11}$ & 0.370 & 0.365 & 0.370 & 0.351 & 0 \\
\hline
\end{tabular}

Taking the bridge condition in 2013 as the present status $C(0)$, the status vector is as shown in (6). The bridge condition during next five years $C(t)$ can be predicted through (7), where $P_{\text {cir }}$ is the Markov transition probability matrix under certain circumstance:

$$
\begin{gathered}
C(0)=[0.565,0.235,0.154,0.036,0.010], \\
C(t)=C(0) \times\left(P_{\text {cir }}\right)^{t}, \quad t=1,2,3,4,5 .
\end{gathered}
$$

The prediction results under three circumstances are displayed by Figures 2, 3, 4, and 5. The results show that the bridge condition will deteriorate rapidly under natural decay circumstance. The condition of the bridge deck system has the fastest decline while the decay rate of the substructure is lowest. It is mainly because the substructure is with the lowest probability of being damaged in urban environment. However, other factors may also lead to this situation such as bridge detection. The substructure is relatively harder to detect because most parts are underwater. At the end of the next five years (2018) the percentage of "A bridges" of deck system will be less than $30 \%$, while the percentage of "E bridges" will be up to $7.8 \%$, which means the number of bridges in danger status will exceed 140. The minor and major repair can mitigate the bridge condition deterioration significantly as shown in the prediction results under conventional recover decay circumstance. It also represents the actual situation of bridge management in Shanghai. Still taking the deck system for example, the percentage of "A bridges" in this situation will be close to $50 \%$ in 2018 , and the "E bridges" will drop to $0.9 \%$. Of course, the results under enhanced recoverable decay are even better. The percentage of "A bridges" will be up to $58 \%$, while the "E bridges" will be reduced to $0.6 \%$, which is even better than the present situation (2013). Although the conditions of bridge deck system and superstructure can be improved under the circumstance of enhanced recoverable decay, as shown in Figures 2(c) and 3(c), it has limited effect on the conditions of substructure and the whole bridge as shown in Figures $4(c)$ and 5(c) compared to the circumstance of conventional recoverable decay.

5.2. Single Bridge Prediction versus Age. Markov-chain model can also be used to predict the condition deterioration versus 


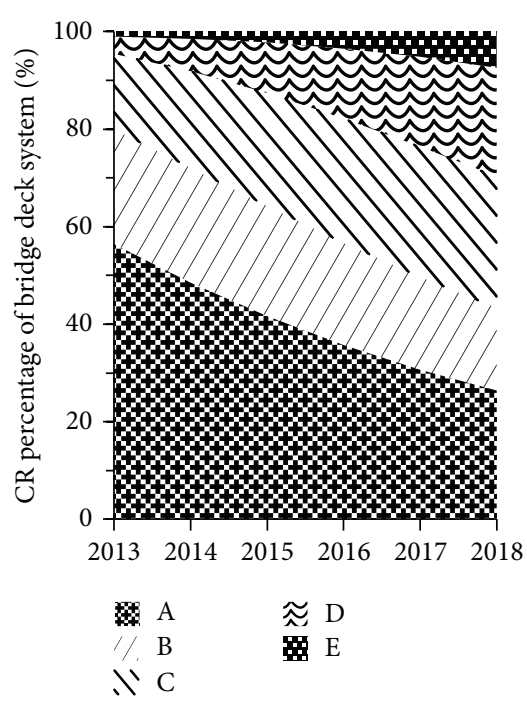

(a) Natural decay
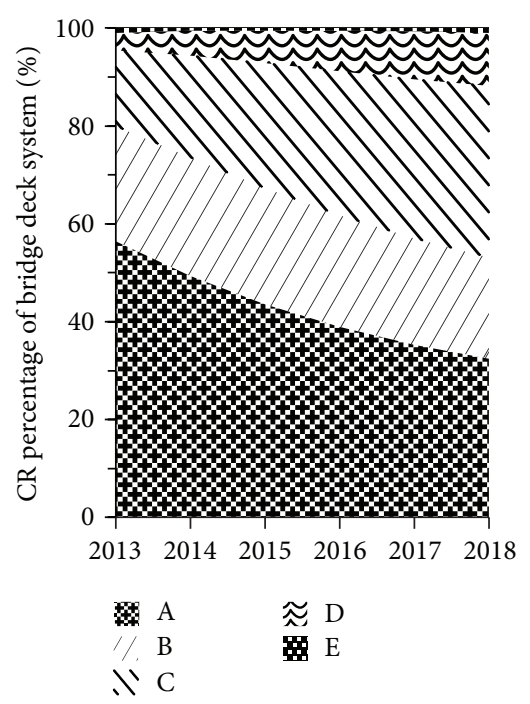

(b) Conventional recoverable decay
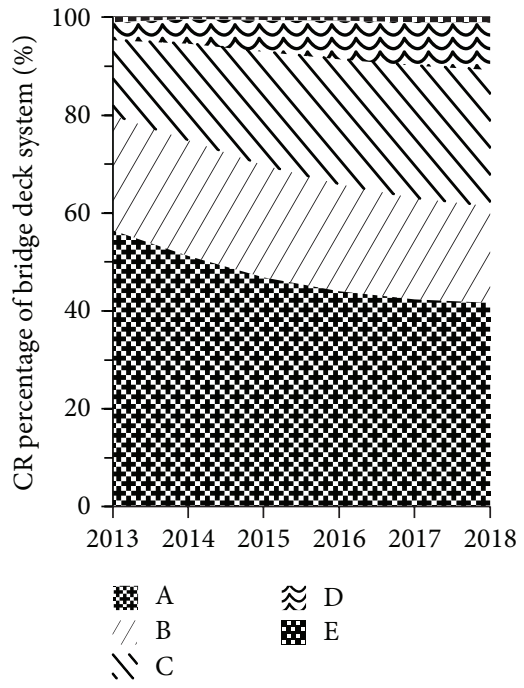

(c) Enhanced recoverable decay

FIGURE 2: Bridge deck system prediction on network level in next five years.

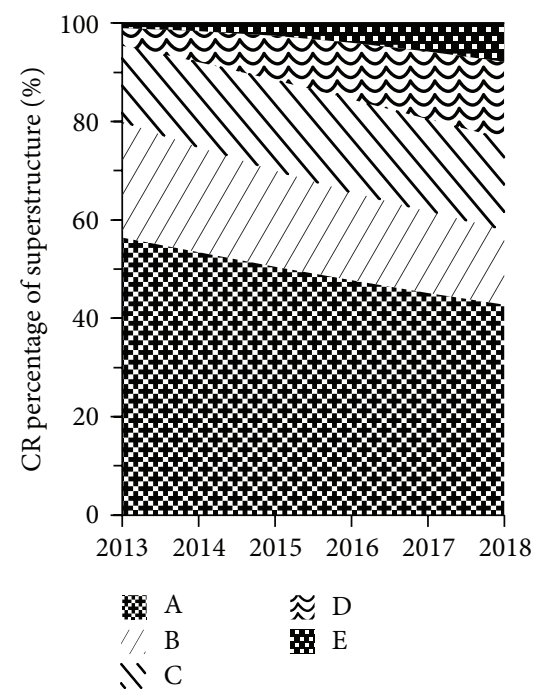

(a) Natural decay
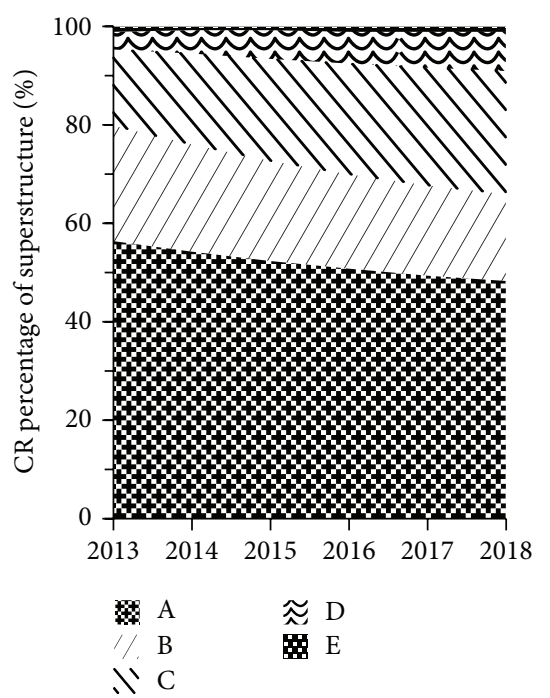

(b) Conventional recoverable decay
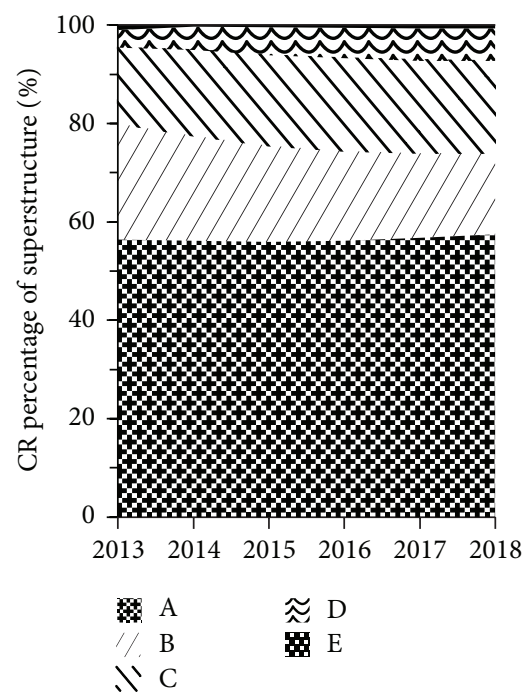

(c) Enhanced recoverable decay

FIGURE 3: Superstructure prediction on network level in next five years.

age of a single bridge under different circumstances. The expected condition can be found in (8), where $C(t)^{*}$ is the condition rating (status value) of single bridge after $t$ years; $C(0)^{*}$ is the status vector of the initial condition of single bridge; $P_{\text {cir }}$ is the transition probability matrix under certain circumstance; and $\mathrm{CR}$ is the bridge condition rating vector, which is constant vector. In this study, $\mathrm{CR}=[5 ; 4 ; 3 ; 2 ; 1]$. Consider

$$
C(t)^{*}=C(0)^{*} \times\left(P_{\text {cir }}\right)^{t} \times \mathrm{CR} .
$$

It is assumed that a bridge deteriorates from the intact state and the forecast period is 20 years. Then the initial status vector $C(0)^{*}$ should be $[1,0,0,0,0]$. The bridge condition deterioration tendency for different components as well as the whole bridge can be found in Figure 6, where the ordinate scales 5 to 1 are equal to CR grades A to $\mathrm{E}$. It is shown that the condition of bridge deck system declines fastest and the CR grade will be below D after 20 years if no recoverable treatments are conducted. Medium and major repair can slow down the deterioration tendency of all bridge components as well as the whole bridge, but it has the greatest effect on the bridge deck system. Enhanced repair has some good influence on the deterioration process, especially in the case of deck system and superstructure, but for the substructure 


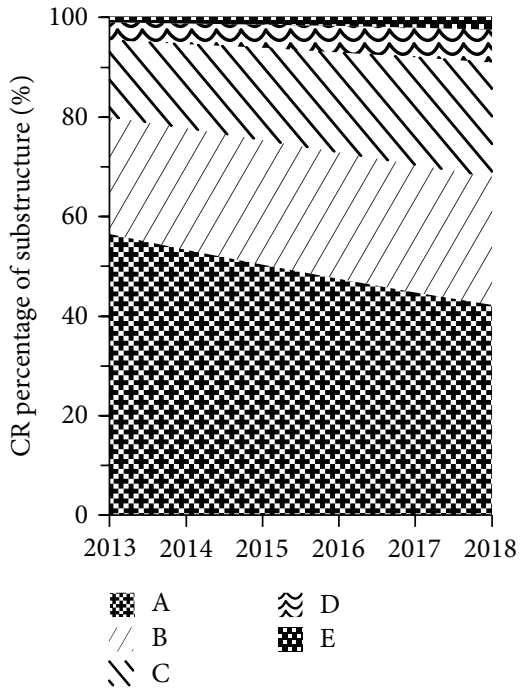

(a) Natural decay

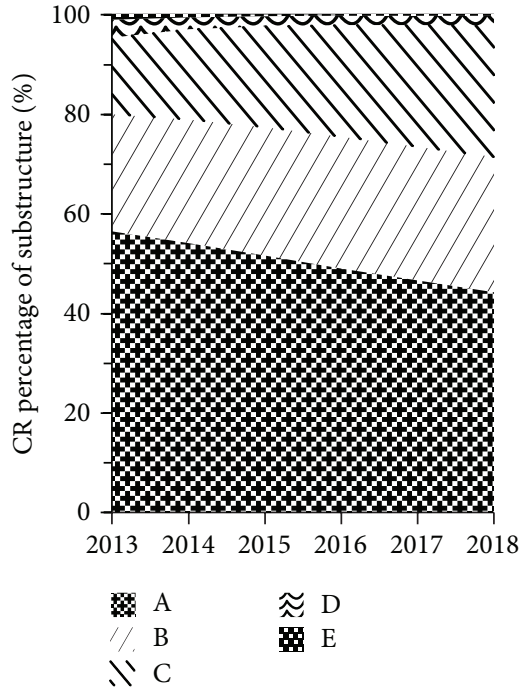

(b) Conventional recoverable decay

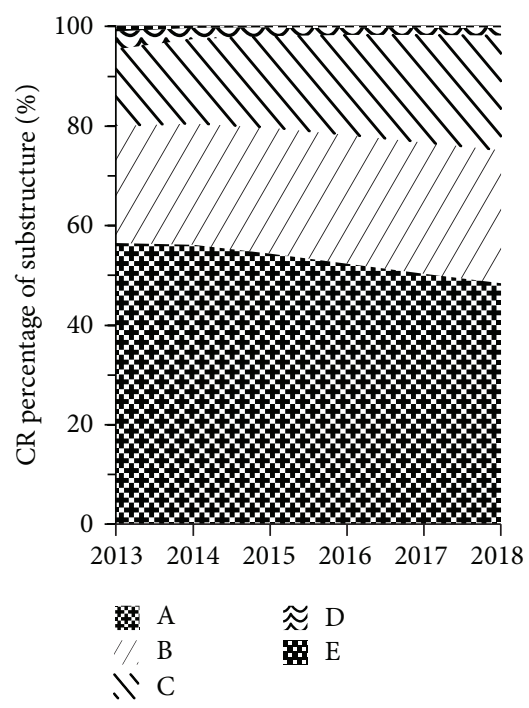

(c) Enhanced recoverable decay

FIGURE 4: Substructure prediction on network level in next five years.

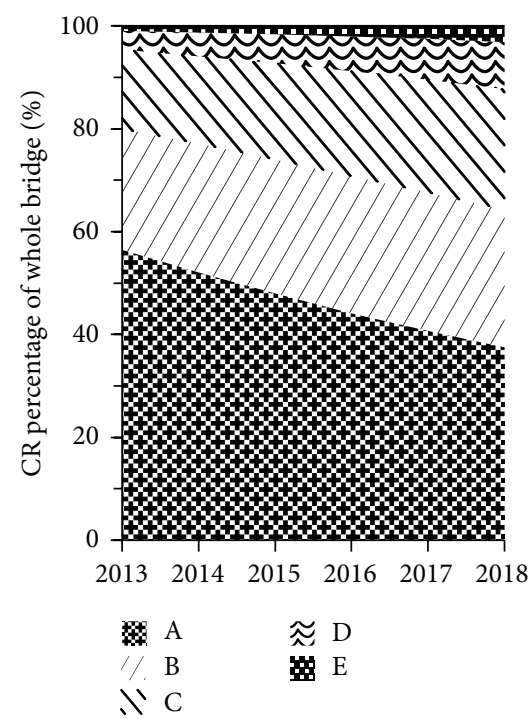

(a) Natural decay
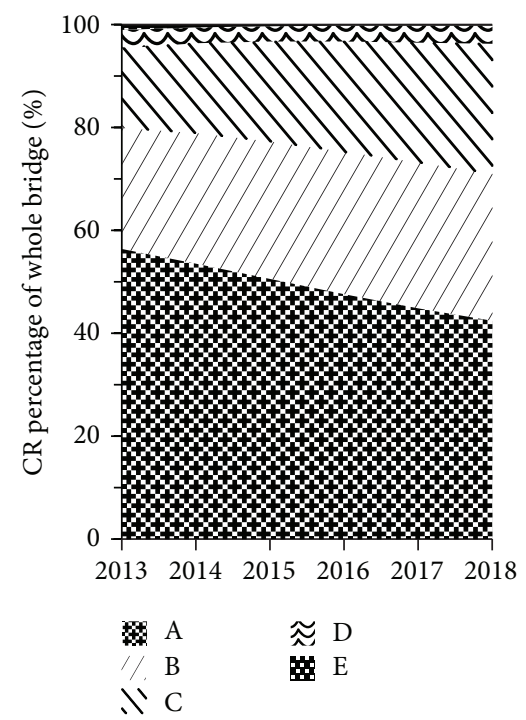

(b) Conventional recoverable decay

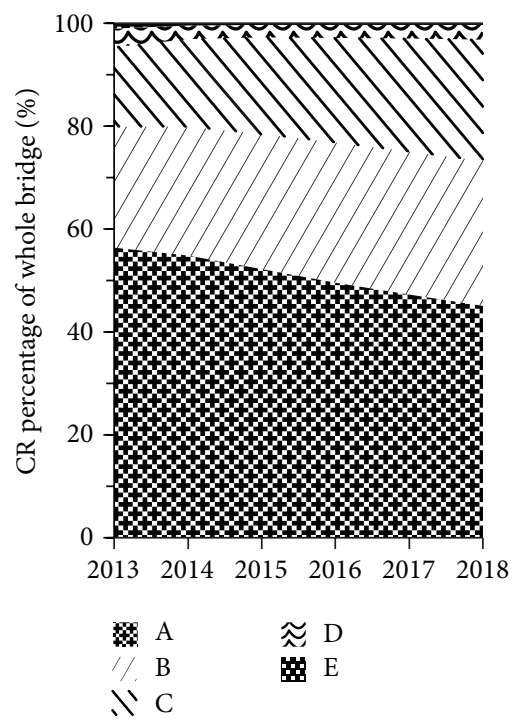

(c) Enhanced recoverable decay

FIGURE 5: Whole bridge prediction on network level in next five years.

and the whole bridge, effect of the enhanced repair is quite little, which means that the present management strategy for urban bridges in Shanghai is reasonable.

\section{Conclusion}

The urban bridge BMS in Shanghai has successfully operated for more than ten years. Thousands of bridge condition data records can be used to predict the deterioration tendency of urban bridges to provide advice for bridge management. However, the basic condition of the data records is complicated because all kinds of bridge status coexist in the same database including bridges without any repair and bridges with different degrees of repair efforts such as routine maintenance, minor, medium and major repair, and even reconstruction. It follows that a prediction model capable of considering a variety of factors should be used to forecast the bridge deterioration process. Therefore a Markov-chain model was proposed in this study. A group of bridge condition data about ten years were used to verify the model and also to find the deterioration tendency of urban bridges in Shanghai.

The CR grades of bridges were used to generate the state vector space and the time step was set to one year because the bridge detection was conducted once a year, which also helped to simplify the form of Markov transition probability 


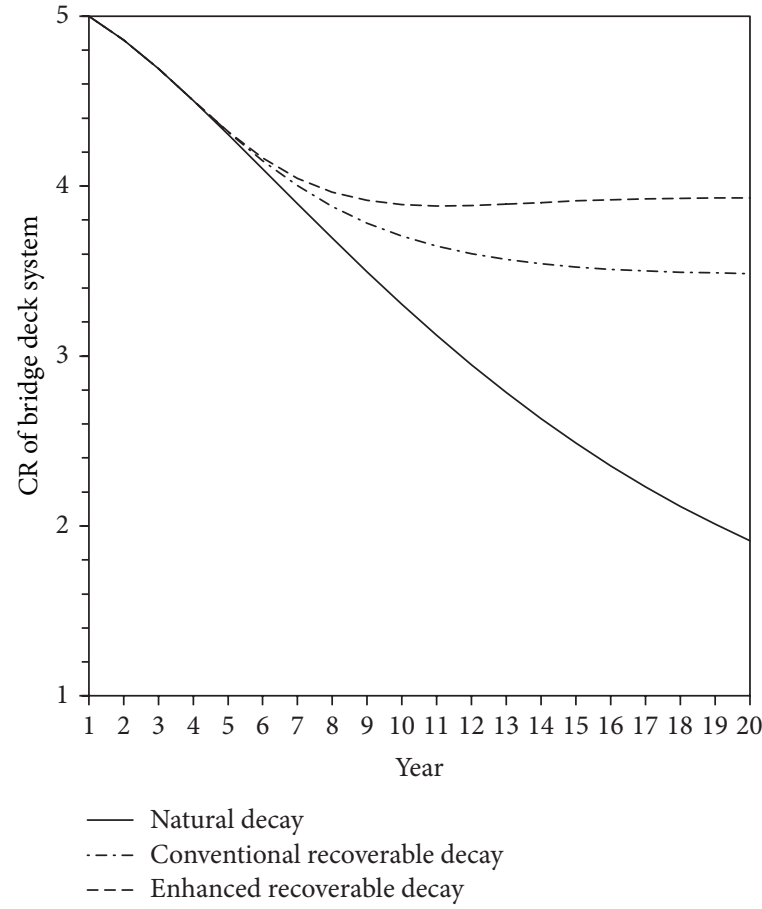

(a) Bridge deck system

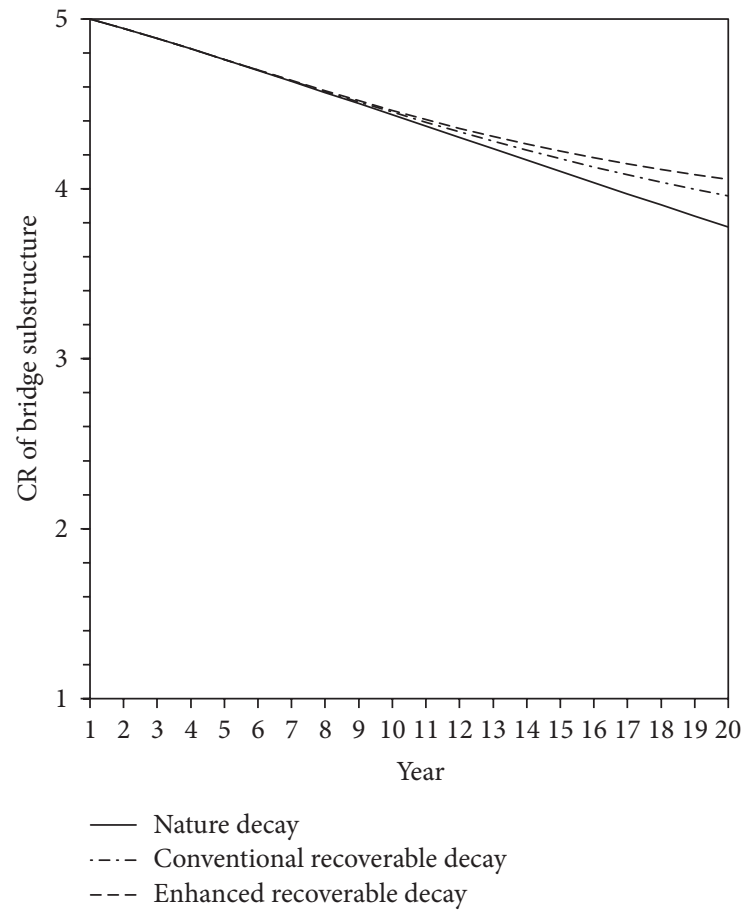

(c) Substructure

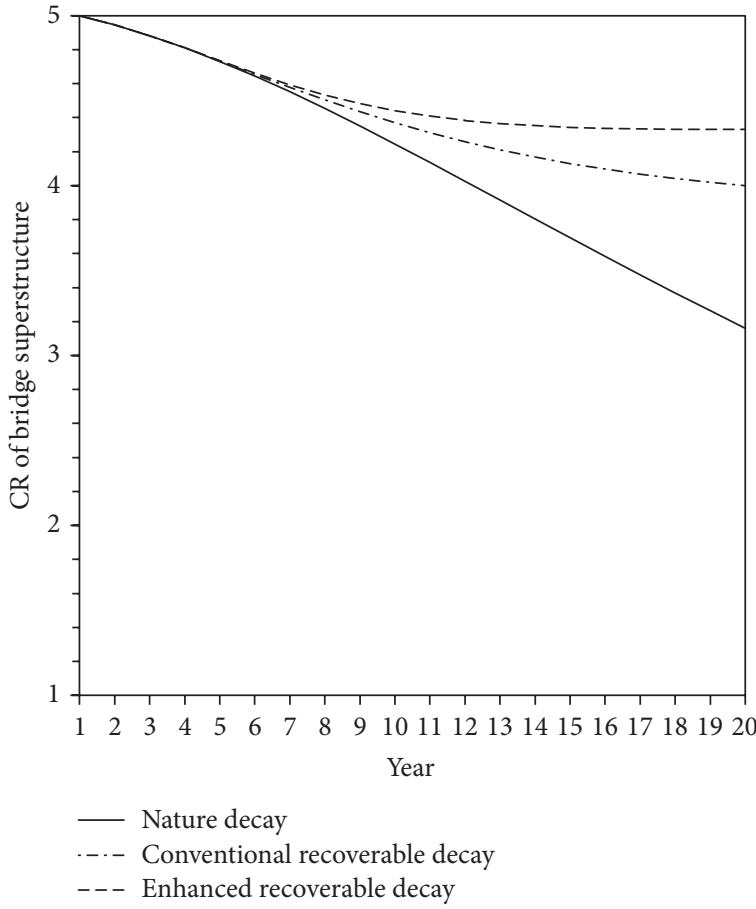

(b) Superstructure

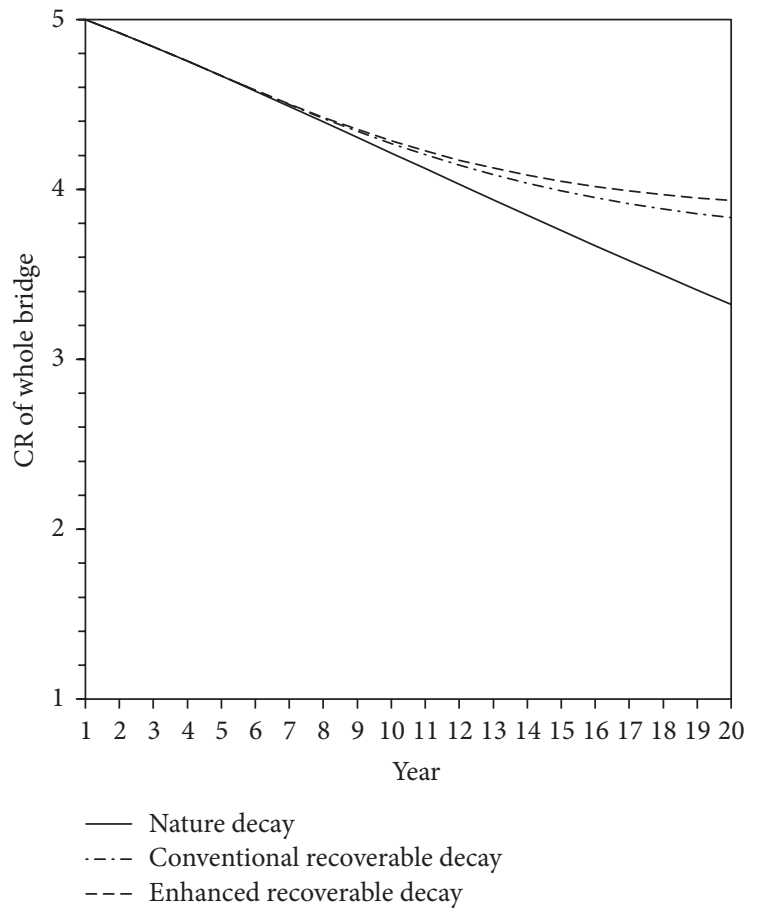

(d) Whole bridge

FIGURE 6: CR deterioration of single bridge.

matrix. The Markov-chain mode can model not only the distribution of the percentage of different CR grades in a transportation network in any year but also the deterioration tendency of a single bridge from any state.

Three decay circumstances were considered in this study, natural decay, conventional recoverable decay, and enhanced recoverable decay. The conventional recoverable decay represents the actual situation of urban bridge management in Shanghai at present, under which only "D and E bridges" need to be repaired to recover to better conditions, while other bridges are under routine maintenance and minor repair so the CR grade cannot be raised. The natural decay is the status 
without any effective maintenance and repair, and CR grades of all bridges in this status cannot be improved. The circumstance of enhanced recoverable decay is an ideal status which fully complies with the Chinese technical regulations of urban bridge management [1], which requires that all "E bridges" and most of "D bridges" should be repaired. Three types of bridge components as well as the whole bridge were predicted by Markov-chain model under the three circumstances mentioned above. The results showed that the bridge conditions would drop rapidly if there were no recoverable repair treatments conducted, especially for the bridge deck system and the superstructure. Proper repair could slow down the deterioration speed of all kinds of bridge components. The effect was most obvious on the bridge deck system and the superstructure came second. The enhanced repair could significantly mitigate the deterioration process and even raise the CR grades after several years of maintenance and repair in the case of the bridge deck system and the superstructure. However other components as well as the whole bridge were not so sensitive to enhanced repair. It seemed the conventional repair was proper enough for the bridges in Shanghai at present and the management strategy applied now was quite reasonable.

\section{Conflict of Interests}

The authors declare that there is no conflict of interests regarding the publication of this paper.

\section{Acknowledgment}

This work was supported by the Postdoctoral Foundation of Jiangxi province in China (2012RC17 and 2013KY32).

\section{References}

[1] “Technical code of maintenance for city bridges," China, CJJ 992003.

[2] Z. Chen, Research on technology structure of transportation infrastructure management system [Ph.D. thesis], Tongji University, Shanghai, China, 2005.

[3] D. Veshosky, C. R. Beildleman, and G. W. Buetow, "Comparative analysis of bridge superstructure deterioration," Journal of Structural Engineering, vol. 120, no. 7, pp. 2123-2136, 1994.

[4] B. Yanev and X. Chen, "Life-cycle performance of New York City bridges," Transportation Research Record 1389, 1993.

[5] S. Ranjith, S. Setunge, R. Gravina, and S. Venkatesan, "Deterioration prediction of timber bridge elements using the Markov chain," Journal of Performance of Constructed Facilities, vol. 27, no. 3, pp. 319-325, 2013.

[6] D. S. Wang, M. Zhu, and J. R. Zhong, "Aided seismic damage prediction system for bridges using statistical analysis methods," World Earthquake Engineering, vol. 19, no. 3, pp. 117-122, 2003.

[7] S. Madanat, R. Mishalani, and W. H. W. Ibrahim, "Estimation of infrastructure transition probabilities from condition rating data," Journal of Infrastructure Systems, vol. 1, no. 2, pp. 120-125, 1995.

[8] J. L. Bogdanoff, "A new cumulative damage model-part I," Journal of Applied Mechanics, vol. 45, no. 2, pp. 246-250, 1978.
[9] T. Micevski, G. Kuczera, and P. Coombes, "Markov model for storm water pipe deterioration," Journal of Infrastructure Systems, vol. 8, no. 2, pp. 49-56, 2002.

[10] G. Morcous, "Performance prediction of bridge deck systems using markov chains," Journal of Performance of Constructed Facilities, vol. 20, no. 2, pp. 146-155, 2006.

[11] Z. Lounis, "Reliability-based life prediction of aging concrete bridge decks," in Proceedings of the International RILEM Workshop on Life Prediction and Aging Management of Concrete Structures, pp. 229-238, Cannes, France, 2000.

[12] G. Morcous and H. Rivard, "Computer assistance in managing the maintenance of low-slope roofs," Journal of Computing in Civil Engineering, vol. 17, no. 4, pp. 230-242, 2003.

[13] K. Golabi and R. Shepard, "Pontis: a system for maintenance optimization and improvement of US bridge networks," Interfaces, vol. 27, no. 1, pp. 71-88, 1997.

[14] H. Hawk and E. P. Small, "The BRIDGIT bridge management system," Structural Engineering International, vol. 8, no. 4, pp. 309-314, 1998.

[15] M. Cesare, C. Santamarina, C. Turkstra, and E. Vanmarcke, "Modeling bridge deterioration with Markov chains," Journal of Transportation Engineering, vol. 118, no. 6, pp. 820-833, 1992.

[16] E. Parzen, Stochastic Processes, Holden-Day Series in Probability and Statistics, Holden-Day, San Francisco, Calif, USA, 1962.

[17] L. Collins, An Introduction to Markov Chain Analysis, Concepts and Techniques in Modern Geography, Geo Abstracts, 1975.

[18] A. A. Butt, M. Y. Shahin, K. J. Feighan, and S. H. Carpenter, "Pavement performance prediction model using the Markov process," Transportation Research Record, pp. 12-19, 1987.

[19] J. Yi, M. Saito, and K. C. Sinha, "Bridge performance prediction model using the Markov chain," Transportation Research Record, no. 1180, pp. 25-32, 1988. 


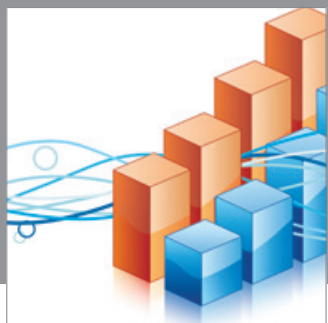

Advances in

Operations Research

mansans

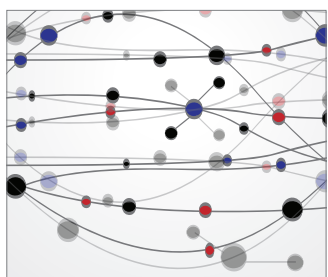

The Scientific World Journal
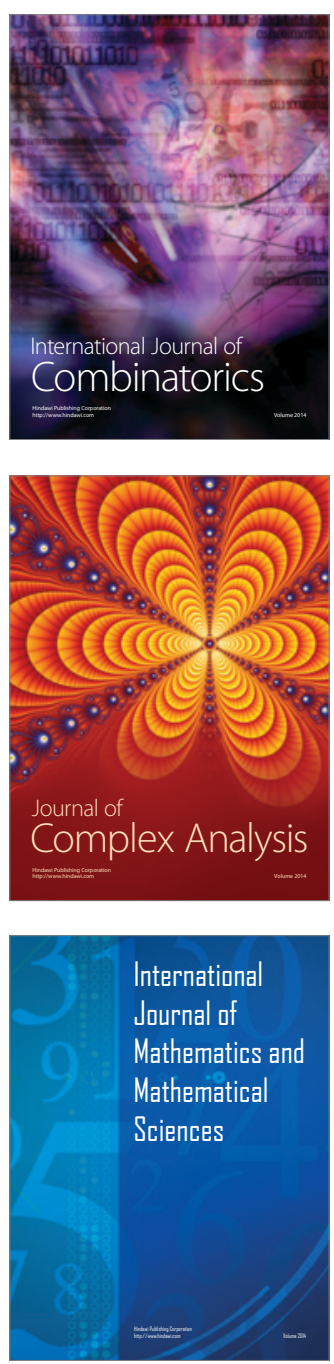
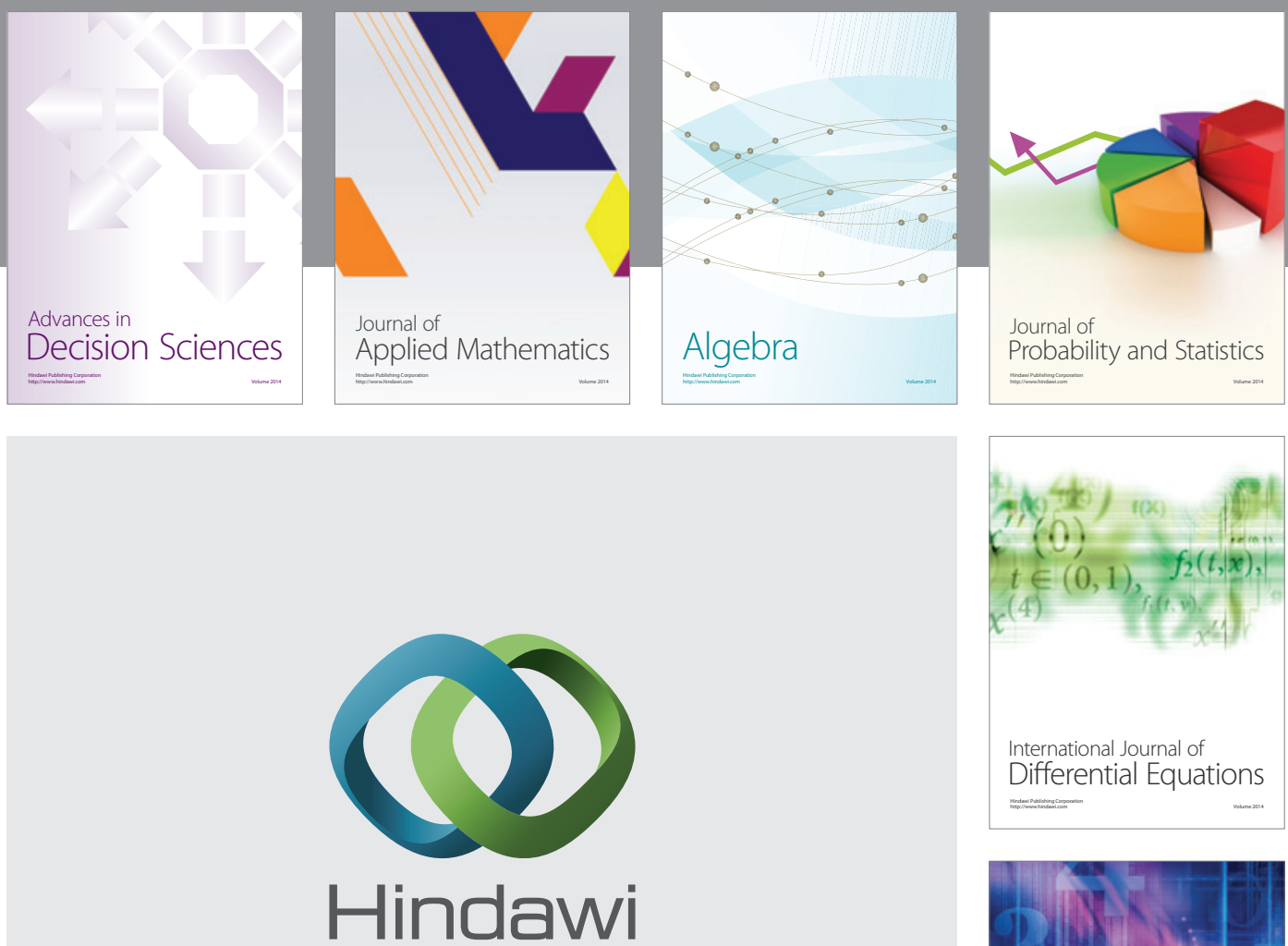

Submit your manuscripts at http://www.hindawi.com
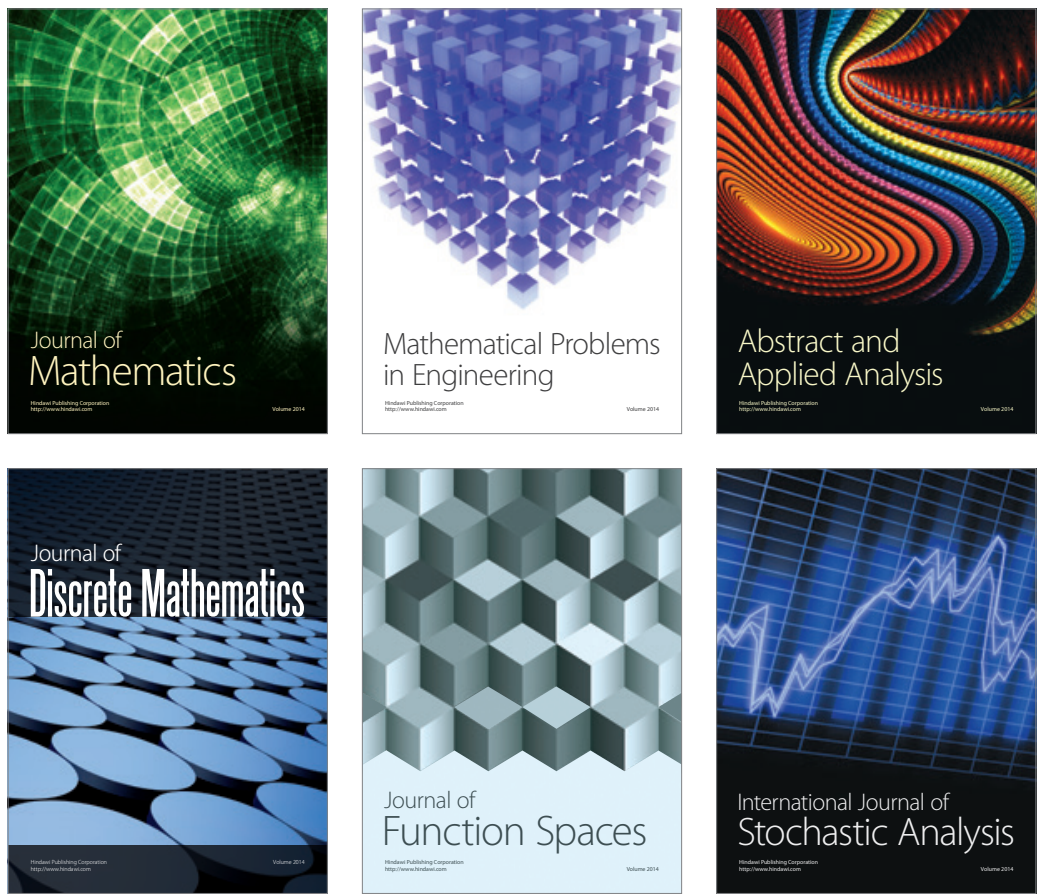

Journal of

Function Spaces

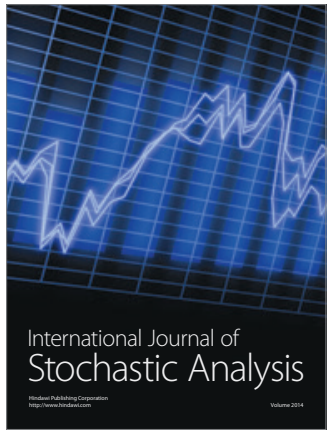

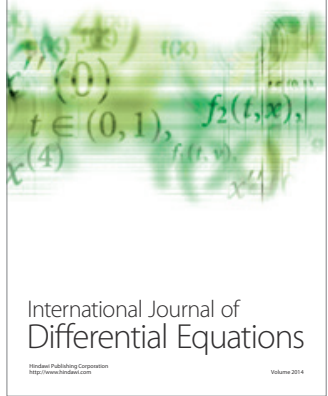
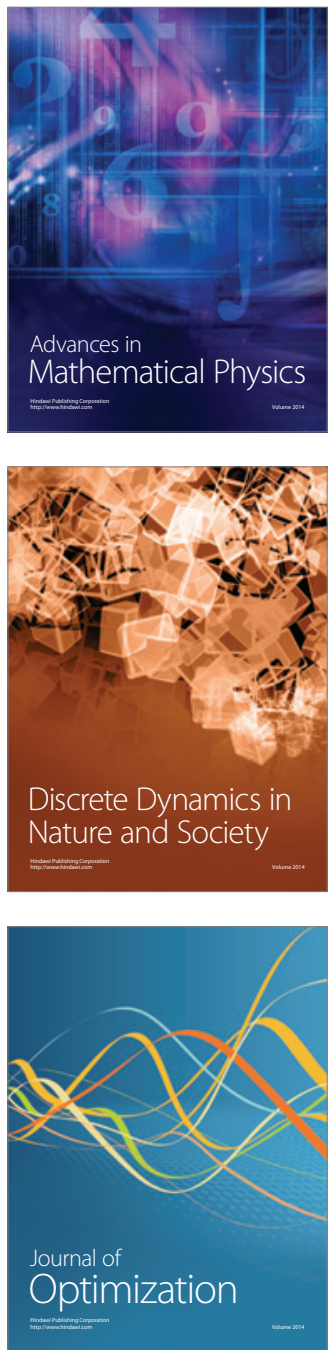